\title{
Dielectric Relaxation, Local Structure and Lattice Dynamics in Mn-Doped Potassium Tantalate Ceramics
}

\author{
Alexander Tkach ${ }^{1, *(\mathbb{D}}$, Abilio Almeida ${ }^{2}$, Igor Levin ${ }^{3}$, Joseph C. Woicik ${ }^{3}$ and Paula M. Vilarinho ${ }^{1}$ \\ 1 Department of Materials and Ceramic Engineering, CICECO-Aveiro Institute of Materials, \\ University of Aveiro, 3810-193 Aveiro, Portugal; paula.vilarinho@ua.pt \\ 2 Department of Physics of Science Faculty, IFIMUP, University of Porto, 4169-007 Porto, Portugal; \\ amalmeid@fc.up.pt \\ 3 Materials Measurement Science Division, National Institute of Standards and Technology, \\ Gaithersburg, MD 20899, USA; igor.levin@nist.gov (I.L.); woicik@bnl.gov (J.C.W.) \\ * Correspondence: atkach@ua.pt
}

Citation: Tkach, A.; Almeida, A.;

Levin, I.; Woicik, J.C.; Vilarinho, P.M. Dielectric Relaxation, Local Structure and Lattice Dynamics in Mn-Doped Potassium Tantalate Ceramics. Materials 2021, 14, 4632. https:// doi.org/10.3390/ma14164632

Academic Editor: Csaba Balázsi

Received: 28 July 2021

Accepted: 12 August 2021

Published: 17 August 2021

Publisher's Note: MDPI stays neutral with regard to jurisdictional claims in published maps and institutional affiliations.

Copyright: (C) 2021 by the authors. Licensee MDPI, Basel, Switzerland. This article is an open access article distributed under the terms and conditions of the Creative Commons Attribution (CC BY) license (https:// creativecommons.org/licenses/by/ $4.0 /)$.

\begin{abstract}
Alkaline niobate and tantalate perovskites have attracted attention as polar dielectrics for electronics and telecommunications. Here, we studied the polar behaviour, lattice dynamics, and local structure in conventionally processed $\mathrm{K}_{0.985} \mathrm{Mn}_{0.015} \mathrm{TaO}_{3 \pm \delta}$ ceramics using a combination of variable-temperature dielectric and Raman spectroscopies, and X-ray absorption fine structure (XAFS) measurements, respectively. Mn doping induces a low-frequency dielectric relaxation in $\mathrm{KTaO}_{3}$ (KT), which follows the Arrhenius law with an activation energy $U \approx 105 \mathrm{meV}$ and the characteristic relaxation time $\tau_{0} \approx 4.6 \times 10^{-14} \mathrm{~s}$. Our XAFS results support preferential Mn occupancy of the cuboctahedral sites as $\mathrm{Mn}^{2+}$, with these cations strongly off-centred in the oversized oxygen cages. Such disordered Mn displacements generate electric dipoles, which are proposed as the source of the observed dielectric relaxation. We show that in Mn-doped ceramics, the low-frequency polar TO1 mode softens on cooling and, at low temperatures, exhibits a higher frequency than in undoped KT. This mode displays no detectable splitting, which contrasts with Li-doped KT that also contains off-centred $\mathrm{Li}^{+}$species on the cuboctahedral sites. Therefore, we conclude that the coupling between the Mn displacements and the lattice is weaker than in the Li case, and Mn-doped KT therefore exhibits a dielectric relaxation but no ferroelectric transition.
\end{abstract}

Keywords: electroceramics; perovskites; polar dielectrics; X-ray absorption; Raman spectroscopy

\section{Introduction}

Perovskite-structured compounds $\mathrm{SrTiO}_{3}(\mathrm{ST}), \mathrm{KTaO}_{3}(\mathrm{KT})$ and $\mathrm{CaTiO}_{3}(\mathrm{CT})$ stand out as incipient ferroelectrics, with their dielectric permittivity increasing continuously on cooling due to the polar-mode softening but without a ferroelectric phase transition [1]. Incipient ferroelectrics exhibit strong dependence of the real part of the dielectric permittivity, $\varepsilon^{\prime}$, on electric field and small values of the dissipation factor, tan $\delta$, which makes them attractive for applications in tunable electronic components [2,3]. Among such $\mathrm{ABO}_{3}$ systems, however, only KT retains an ideal cubic perovskite structure with $P m-3 m$ symmetry down to $0 \mathrm{~K}[4]$.

$\mathrm{Mn}$ is a common dopant in transition metal oxides. The mechanisms for its accommodation in host lattices of perovskite incipient ferroelectrics has been studied most extensively for $\mathrm{SrTiO}_{3}$ [5]. Depending on substitution formulations (i.e., for Sr or Ti) and annealing conditions (temperature, partial oxygen pressure), Mn has been shown to adopt mainly $\mathrm{Mn}^{4+}$ and $\mathrm{Mn}^{2+}$ oxidation states. While ST systems containing exclusively $\mathrm{Mn}^{4+}$ could be obtained, $\mathrm{Mn}^{2+}$ has always coexisted with $\mathrm{Mn}^{4+}$ or $\mathrm{Mn}^{3+}$. The site preference for $\mathrm{Mn}$ varies with its oxidation state and the resulting effective ionic radius. The smaller $\mathrm{Mn}^{4+}$ species always occupy octahedral B sites, whereas the significantly larger $\mathrm{Mn}^{2+}$ cations prefer cuboctahedral A sites [5]. A recent study of Mn doping in CT revealed 
dissimilar behaviour compared to that in Mn-doped ST. In CT, Mn is present in the mixed $\mathrm{Mn}^{4+} / \mathrm{Mn}^{2+}$ state regardless of its substitution for $\mathrm{Ca}$ or $\mathrm{Ti}$ in the formula, with $\mathrm{Mn}^{4+}$ and $\mathrm{Mn}^{2+}$ cations located in the octahedral and cuboctahedral sites, respectively [6]. In both ST- and CT-based systems, $\mathrm{Mn}^{2+}$ species are strongly off-centred in the oversized cuboctahedral cages, generating electric dipoles. The presence of such positional disorder for $\mathrm{Mn}^{2+}$ has been invoked as an explanation for the dielectric relaxation and multi-glass (i.e., dipolar plus spin glass) behaviour observed in Mn-doped ST [7]. The multi-glass state has been associated with polar displacements of $\mathrm{Mn}^{2+}$ in the highly polarizable incipient ferroelectric lattice, thereby initiating a transition of the $\mathrm{Mn}^{2+}$ magnetic moments into a spin glass.

KT-based compounds have mainly been investigated as single crystals [8,9]. Although ceramics are assumed to possess higher and more controllable dopant contents, being also cheaper to fabricate than single crystals, the scarcity of works on ceramics can be attributed to the relatively low melting temperature of $\mathrm{KT}$, which, combined with the high volatility of potassium, makes it challenging to synthesize dense monophasic ceramics with well-controlled chemical compositions [10]. Among KT-based compounds, $\mathrm{K}_{1-x} \mathrm{Li}_{x} \mathrm{TaO}_{3}$ solid solutions have been studied intensively, wherein displacements of small $\mathrm{Li}^{+}$ions on K sites generate strong local dipole moments that couple electrostatically to the KT's polar soft mode [8,9]. As a result, both dielectric relaxations and a ferroelectric phase transition were reported for the $\mathrm{K}_{1-x} \mathrm{Li}_{x} \mathrm{TaO}_{3}$ system [8,9,11-13]. Similar to Li-doped KT and Mn-doped ST, Mn-doped (0.01\% to 0.3\%) KT single crystals [14-16] exhibit a dielectric relaxation which has also been attributed to a positional disorder of $\mathrm{Mn}^{2+}$ on $\mathrm{K}$ sites [15].

Mn-doped KT ceramics [17-19] feature a relaxation at low frequencies, while in the microwave range, the relaxational effects are manifested in $\tan \delta$, but not in $\varepsilon^{\prime}$ [19-21]. The $\mathrm{K}_{0.97} \mathrm{Mn}_{0.03} \mathrm{TaO}_{3 \pm \delta}$ ceramics $[17,18]$ display both dielectric and magnetic anomalies, which resemble the "multi-glass" behaviour observed in Mn-doped ST [7,22]. However, the interpretation of the magnetic response in $\mathrm{K}_{0.97} \mathrm{Mn}_{0.03} \mathrm{TaO}_{3 \pm \delta}$ is complicated because of a contribution from the second phase, $\mathrm{MnO}_{y}$. Indeed, $\mathrm{MnO}_{y}$ has been detected in elemental maps of $\mathrm{K}_{0.97} \mathrm{Mn}_{0.03} \mathrm{TaO}_{3 \pm \delta}$ ceramics, whereas $\mathrm{K}_{0.985} \mathrm{Mn}_{0.015} \mathrm{TaO}_{3 \pm \delta}$ and $\mathrm{KTaO}_{3}$ have displayed homogeneous elemental distributions devoid of chemical segregation while featuring distinct lattice parameters [23]. A study of the magnetic response in $\mathrm{K}_{1-2 x} \mathrm{Mn}_{x} \mathrm{TaO}_{3}$ loose powders, which have been formulated with the intentional $\mathrm{K}$ deficiency to provide charge compensation for the aliovalent $\mathrm{Mn}^{2+}$ substitution, claimed the formation of $\mathrm{Mn}_{3} \mathrm{O}_{4}$ for $x \geq 0.01$ [24]. However, the same group reported contradictory data on the Mn solubility limit for this formulation by demonstrating a linear dependence of the lattice parameter on Mn content up to $x=0.03$ [20]. For $x=0.04$, a Ta-rich tungsten bronze structure was detected as the main extra phase [20]. In addition to this apparent controversy regarding the Mn solubility limit in the loose powders $[20,24], \mathrm{K}_{1-2 x} \mathrm{Mn}_{x} \mathrm{TaO}_{3}$ ceramics exhibited a non-monotonic trend for the dielectric relaxation, with the relaxation strength being the strongest for $x=0.01$, significantly diminished for $x=0.02$ and 0.03 , and partially restored for $x=0.05[19,24]$. Such an inconsistent behaviour of the relaxation suggests that for $x \geq 0.02, \mathrm{~K}_{1-2 x} \mathrm{Mn}_{x} \mathrm{TaO}_{3}$ ceramics contain other phases. Overall, the available data indicate that for the $\mathrm{K}_{1-2 x} \mathrm{Mn}_{x} \mathrm{TaO}_{3}$ nominal stoichiometry, a controlled Mn substitution is difficult to achieve.

Thus far, little research has been undertaken into the local structure and lattice dynamics in Mn-doped KT ceramics or single crystals, especially as a function of temperature. Here, we combined variable-temperature dielectric measurements over a broad frequency range, room-temperature X-ray absorption fine structure (XAFS) measurements, and variable-temperature Raman spectroscopy to determine the site occupancy and coordination environments for the Mn dopant species in KT ceramics that have been confirmed as monophasic [23]. 


\section{Materials and Methods}

For this study, we selected ceramics with the nominal $\mathrm{K}_{0.985} \mathrm{Mn}_{0.015} \mathrm{TaO}_{3 \pm \delta}$ composition prepared using conventional solid-state synthesis, which has been demonstrated to yield a single perovskite phase [23]. The $\mathrm{K}_{2} \mathrm{CO}_{3}$ (Merck KGaA, Darmstadt, Germany, purity $99+\%$ ), $\mathrm{Mn}\left(\mathrm{NO}_{3}\right)_{2}$ (Merck KGaA, Darmstadt, Germany, purity $98.5+\%$ ) and $\mathrm{Ta}_{2} \mathrm{O}_{5}$ (SigmaAldrich, Saint Louis, MO, USA, purity 99+\%) reagents (Certain commercial products or company names are identified here to describe our study adequately, not intending to imply recommendation or endorsement by National Institute of Standards and Technology (NIST), nor is it intended to imply that the products or names identified are necessarily the best available for the purpose.) were dried to remove moisture, mixed, and milled in alcohol for $5 \mathrm{~h}$ in a planetary mill using Teflon containers and zirconia spherical grinding media. The loss of potassium, which is expected to occur during sintering, was compensated by adding $5 \mathrm{wt} . \%$ excess of potassium to the initial mixture prior to the milling [10]. The resulting powders were dried and calcined at $875{ }^{\circ} \mathrm{C}$ for $8 \mathrm{~h}$. The calcined powders were milled again for $5 \mathrm{~h}$ to reduce the particle size below $5 \mu \mathrm{m}$, and uniaxially pressed $(100 \mathrm{MPa})$ into pellets, $10 \mathrm{~mm}$ in diameter. The pellets, covered with powder of the same composition to mitigate the loss of potassium, were sintered in closed alumina crucibles in air at $1350{ }^{\circ} \mathrm{C}$ for $1 \mathrm{~h}$ with a heating and cooling rate of $5{ }^{\circ} \mathrm{C} / \mathrm{min}$, similarly to the conditions used previously for preparing undoped and Li-doped KT ceramics [11]. After sintering, the pellets exhibited a density of $\approx 88 \%$ and an average grain size of $\approx 1.8 \mu \mathrm{m}$.

For dielectric measurements, gold electrodes were sputtered on both sides of the polished ceramic pellets. Complex dielectric permittivity, including its real $\varepsilon^{\prime}$ and imaginary $\varepsilon^{\prime \prime}$ parts, were measured in the frequency range from $10^{2} \mathrm{~Hz}$ to $10^{8} \mathrm{~Hz}$ between room temperature and $10 \mathrm{~K}$ using a precision LCR-meter (HP 4284A, Hewlett Packard, Palo Alto, USA) and a RF Impedance Analyzer (HP 4191A, Hewlett Packard, Palo Alto, CA, USA). The dissipation factor was calculated as $\tan \delta=\varepsilon^{\prime \prime} / \varepsilon^{\prime}$. Unpolarised Raman spectra were recorded in the spectral range from $10 \mathrm{~cm}^{-1}$ to $1000 \mathrm{~cm}^{-1}$ in a pseudo-backscattering geometry using a spectrometer (Jobin-Yvon T64000, Horiba, Kyoto, Japan) equipped with a charge-coupled device and a photon-counting detector. The spectral slit width was about $1.5 \mathrm{~cm}^{-1}$, while an excitation was performed using a Spectra Physics argon laser operating at a wavelength $\lambda=514.5 \mathrm{~nm}$. The spectra were registered at a series of temperatures between $10 \mathrm{~K}$ to $290 \mathrm{~K}$, after a waiting time of $15 \mathrm{~min}$ at each temperature. During the dielectric and Raman-spectroscopy measurements, temperature $(T)$ control was achieved using a He closed-cycle cryogenic system (Displex APD-Cryostat HC-2, Allentown, PA, USA), equipped with silicon diode temperature sensors and a digital temperature controller Scientific Instruments Model 9650. XAFS measurements for the Mn K-edge (6539 eV) were conducted at ambient temperature at the NIST X23A2 beamline of the National Synchrotron Light Source (NSLS, Brookhaven National Laboratory) on finely ground (mortar and pestle) powders dispersed on a double-sided scotch tape. The double-crystal monochromator was operated with a pair of $\mathrm{Si}(311)$ crystals. About 50 spectra were collected in the fluorescence mode using a four-element Si-drift detector. The detector count rate was adjusted to minimize the dead-time effects [25]. Portions of X-ray absorption spectra containing nearedge (XANES) and extended (EXAFS) fine structure were recorded in the same run using smaller energy steps for XANES and longer counting times for EXAFS. Transmission data from a Mn foil positioned downstream of the sample was recorded simultaneously with each scan for energy calibration. Mn spectra for the reference samples of $\mathrm{SrMn}^{4+} \mathrm{O}_{3}$, $\mathrm{YMn}^{3+} \mathrm{O}_{3}$, and $\mathrm{Mn}^{2+} \mathrm{TiO}_{3}$ have been measured previously at the same beamline. These measurements were performed on powder samples in transmission. The XAFS data were processed and analysed using Athena and Artemis software, respectively [26]. Scattering phases and amplitudes were calculated using FEFF8 [27].

\section{Results and Discussion}

As reported previously, undoped KT ceramics upon cooling display a continuous increase of the dielectric permittivity to $\varepsilon^{\prime} \approx 4000$ without frequency dispersion $[10,11]$. 
In contrast, for the Mn-doped KT ceramics, the maximum attainable value of $\varepsilon^{\prime}$ is only $\approx 1400$ (see Figure 1a), and the $\varepsilon^{\prime}(T)$ dependence reveals a diffuse peak with a frequencydependent amplitude and position, suggesting a dielectric relaxation. This relaxation is also manifested by the frequency-dependent peaks in both $\varepsilon^{\prime \prime}(T)$ (see Figure $1 b$ ) and $\tan \delta(T)$ (see Figure 1c), with the peak-maximum temperature varying from about $50 \mathrm{~K}$ at $100 \mathrm{~Hz}$ to about $116 \mathrm{~K}$ at $100 \mathrm{MHz}$. Our results reconcile the observation of a permittivity peak for frequencies up to $10 \mathrm{MHz}$ by Shvartsman et al. [18] and the absence of such a peak at microwave frequencies between $2.5 \mathrm{GHz}$ and $3.5 \mathrm{GHz}$ by Axelsson et al. [20,21]. Indeed, the peak in $\varepsilon^{\prime}(T)$ becomes invisible above $10 \mathrm{MHz}$, but the corresponding peaks in $\varepsilon^{\prime \prime}(T)$ and $\tan \delta(T)$ are still observed even above this frequency. For the $\mathrm{K}_{0.97} \mathrm{Mn}_{0.03} \mathrm{TaO}_{3 \pm \delta}$ ceramics studied in [18], the dielectric relaxation occurred at approximately the same temperatures as observed here for $\mathrm{K}_{0.985} \mathrm{Mn}_{0.015} \mathrm{TaO}_{3 \pm \delta}$, but it appears to be stronger, which is consistent with a larger number of independent dipoles created for the higher Mn content.

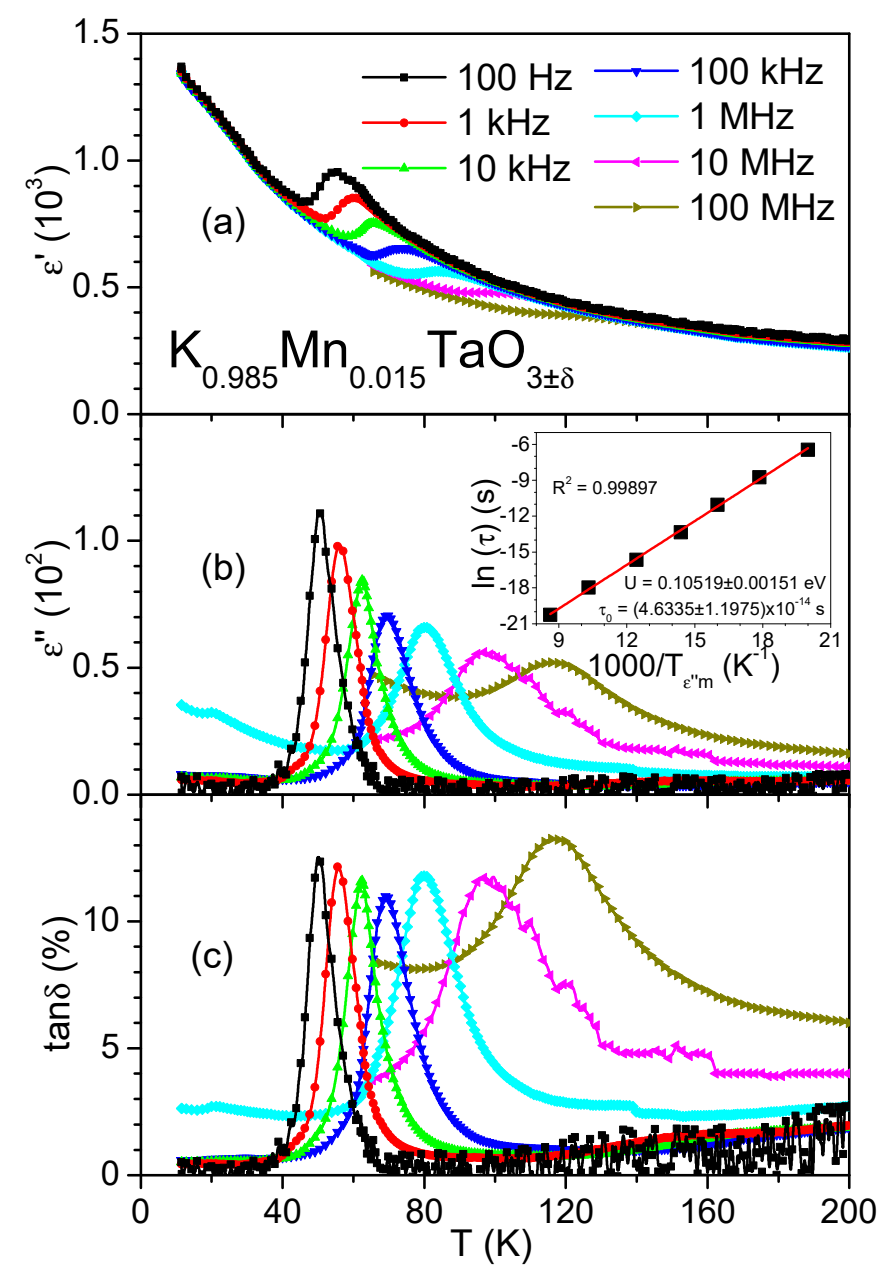

Figure 1. Real $\varepsilon^{\prime}(\mathbf{a})$ and imaginary $\varepsilon^{\prime \prime}(\mathbf{b})$ parts of the dielectric permittivity and dissipation factor $\tan \delta$ (c) of $\mathrm{K}_{0.985} \mathrm{Mn}_{0.015} \mathrm{TaO}_{3 \pm \delta}$ ceramics as a function of temperature $T$ in the $100 \mathrm{~Hz}-100 \mathrm{MHz}$ frequency range. Inset shows the Arrhenius plot $\ln (\tau)$ versus $1000 / T_{\varepsilon^{\prime \prime} \mathrm{m}}$ for the dielectric relaxation of $\mathrm{K}_{0.985} \mathrm{Mn}_{0.015} \mathrm{TaO}_{3 \pm \delta}$ ceramics with fits to the Arrhenius law (solid line) and the fit parameters.

We clarified the origins of electric dipoles responsible for the relaxation by using the Debye approximation to analyse relaxational dynamics from the behaviour of the maximum of the $\varepsilon^{\prime \prime}(T)$ peak as a function of temperature and frequency. In this approach, a set of independent dipoles is characterized by a unique relaxation time $(\tau)$, which is equal to the inverse of the angular relaxation frequency $(\omega=2 \pi f)$. The relaxation is described using the Arrhenius law: 


$$
\tau=\tau_{0} \exp \left(U / \mathrm{k}_{\mathrm{B}} T\right)
$$

where $\tau_{0}$ is the relaxation time at infinite temperature. Here, $U$ is the activation energy of the dipolar process, $\mathrm{k}_{\mathrm{B}}$ is the Boltzmann constant, and $T$ is the absolute temperature. The dynamics of the diffuse peak in the frequency range from $10^{2} \mathrm{~Hz}$ to $10^{8} \mathrm{~Hz}$ can be examined using a plot (inset in Figure $1 b$ ) of $\ln (\tau)$ vs. $1000 / T_{\varepsilon^{\prime \prime} m}$, where $T_{\varepsilon^{\prime \prime} m}$ is the temperature that corresponds to the maximum of the $\varepsilon^{\prime \prime}(T)$ peak at the angular frequency $w=2 \pi f=\tau^{-1}$. From this plot, we find $U=105 \mathrm{meV}$ and $\tau_{0}=4.6 \times 10^{-14} \mathrm{~s}$, which are close to the corresponding values determined for Mn-doped ( $0.01 \%$ to $0.3 \%) \mathrm{KT}$ single crystals using dielectric-spectroscopy and electron-spin-resonance (ESR) measurements [14,15], as shown in Table 1 . Our values of $U$ and $\tau_{0}$ are also close to those reported for Mn-doped $(0.5 \%$ to $5 \%) \mathrm{KT}$ ceramics $[18,19]$ (Table 1$)$, with the caveat that Mn content in some of these compositions exceeded the solubility limit.

Table 1. Arrhenius law parameters for the dielectric relaxation dynamics in $\mathrm{Mn}$-doped $\mathrm{KTaO}_{3}$.

\begin{tabular}{ccccc}
\hline \multirow{2}{*}{ Composition } & \multirow{2}{*}{ State } & \multicolumn{2}{c}{ Arrhenius Law } & \multirow{2}{*}{ Ref. } \\
\cline { 3 - 4 } & & $\boldsymbol{U}$ (meV) & $\boldsymbol{\tau}_{\mathbf{0}}$ (s) & \\
\hline $\mathrm{K}_{0.985} \mathrm{Mn}_{0.015} \mathrm{TaO}_{3 \pm \delta}$ & ceramics & 105 & $4.6 \times 10^{-14}$ & This work \\
$\mathrm{K}_{0.97} \mathrm{Mn}_{0.03} \mathrm{TaO}_{3 \pm \delta}$ & ceramics & 120 & $4.2 \times 10^{-14}$ & {$[18]$} \\
$\mathrm{K}_{1-2 x} \mathrm{Mn}_{x} \mathrm{TaO}_{3}(x=0.5-5 \%)$ & ceramics & $111-118$ & $(4-11) \times 10^{-14}$ & {$[19]$} \\
$\mathrm{KTaO}_{3}: \mathrm{Mn}(0.01-0.1 \%)$ & single crystal & $105-110$ & $2 \times 10^{-14}$ & {$[14]$} \\
$\mathrm{KTaO}_{3}: \mathrm{Mn}(0.01-0.3 \%)$ & single crystal & 110 & $2 \times 10^{-14}$ & {$[15]$} \\
$\mathrm{KTaO}_{3}: \mathrm{Mn}(0.01 \%, \mathrm{ESR})$ & single crystal & 104 & $0.5 \times 10^{-14}$ & {$[15]$} \\
\hline
\end{tabular}

Since a prior microscopic characterization of the present ceramics confirmed a homogenous distribution of $\mathrm{Mn}$ within the KT lattice [23], the observed dielectric relaxation should have intrinsic origins. For (001)-oriented $\mathrm{Mn}$-doped $\mathrm{KTaO}_{3}$ single crystals, studied by dielectric spectroscopy and ESR techniques, the relaxation with the activation energy of 104-110 meV has been proven to originate from polar displacements of $\mathrm{Mn}^{2+}$ cations occupying the $\mathrm{K}$ sites [15]. Although ceramics differ from single crystals, possessing grain boundaries and pores but having no preferred crystallographic orientation, similar activation energies in our ceramics and the previously studied Mn-doped KT single crystals [15] indicate that the dielectric relaxation observed here can be related to the same disordered-ion-displacement mechanism [8,15].

Our XAFS results support this inference, providing evidence for the presence of $\mathrm{Mn}$ on the cuboctahedral sites in $\mathrm{KT}$ as strongly off-centred $\mathrm{Mn}^{2+}$ species. Figure 2 compares XANES for $\mathrm{Mn}$ in $\mathrm{K}_{0.985} \mathrm{Mn}_{0.015} \mathrm{TaO}_{3 \pm \delta}, \mathrm{Mn}^{2+} \mathrm{TiO}_{3}$, and $\mathrm{SrMn}^{4+} \mathrm{O}_{3}$. Clearly, the doped KT ceramics contain a significant fraction of $\mathrm{Mn}^{2+}$. A linear combination fit using $\mathrm{MnTiO}_{3}$ and $\mathrm{SrMnO}_{3}$ as references provides a reasonably good match to the spectrum for the doped KT and therefore suggests that the latter contains a mixture of $\mathrm{Mn}^{2+}$ and $\mathrm{Mn}^{4+}$ cations with the 2:1 ratio. Both XANES and EXAFS (Figure 3a) for $\mathrm{K}_{0.985} \mathrm{Mn}_{0.015} \mathrm{TaO}_{3 \pm \delta}$ are similar to those for the previously studied $\mathrm{Sr}_{0.98} \mathrm{Mn}_{0.02} \mathrm{TiO}_{3}$ ceramics, which have been shown to contain a mixture of $\mathrm{Mn}^{2+}$ and $\mathrm{Mn}^{4+}$ residing on the A- and B-sites, respectively.

Given the mixed oxidation state of Mn suggested by the XANES, we tested two models for the coordination of Mn while fitting the EXAFS data (Figure 3b). Model 1 assumed the presence of $\mathrm{Mn}$ on both octahedral $\left(\mathrm{as} \mathrm{Mn}^{4+}\right)$ and cuboctahedral $\left(\mathrm{Mn}^{2+}\right)$ sites. In contrast, Model 2 considered a mixture of two different octahedral sites populated by the smaller $\mathrm{Mn}^{4+}$ (ionic radius $0.53 \AA$ ) and larger $\mathrm{Mn}^{2+}(0.83 \AA$ [28]) species, respectively. The Mn EXAFS data are insufficient to support reliable refinements of all the distances and their associated Debye-Waller (D-W) factors for the two coordination environments as independent variables [29]. Therefore, we adopted a previously used strategy of fixing most of the structural parameters for the rigid $\mathrm{Mn}^{4+}$ octahedral coordination at their wellcharacterized values for $\mathrm{Mn}^{4+}$ in doped $\mathrm{SrTiO}_{3}$ (see the footnote to Table 2 for more details). 
The parameters describing the coordination of $\mathrm{Mn}^{2+}$ in Models 1 and 2 were treated as variables; a fraction of the $\mathrm{Mn}^{2+}$ species was refined as well. For the K-site coordination, only single-scattering paths of the photoelectron were included in the fit since in this case the contributions of multiple-scattering events are negligible. For the octahedrally coordinated $\mathrm{Mn}$, we considered both single and multiple scattering paths.

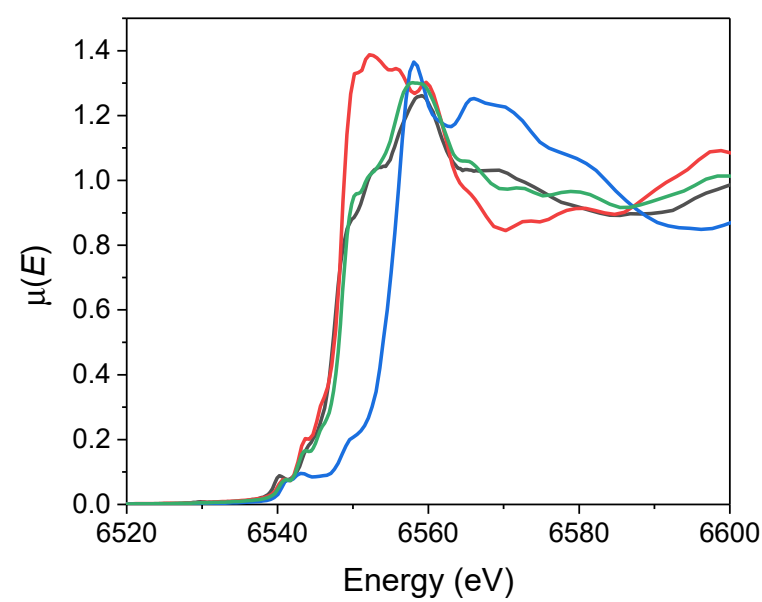

Figure 2. Mn K-edge X-ray absorption near-edge structure (XANES) for $\mathrm{K}_{0.985} \mathrm{Mn}_{0.015} \mathrm{TaO}_{3 \pm \delta}$ (black), $\mathrm{MnTiO}_{3}$ (red), $\mathrm{SrMnO}_{3}$ (blue). A fit of the $\mathrm{K}_{0.985} \mathrm{Mn}_{0.015} \mathrm{TaO}_{3 \pm \delta}$ XANES using a linear combination of the spectra for $\mathrm{MnTiO}_{3}$ and $\mathrm{SrMnO}_{3}$ is shown in green.

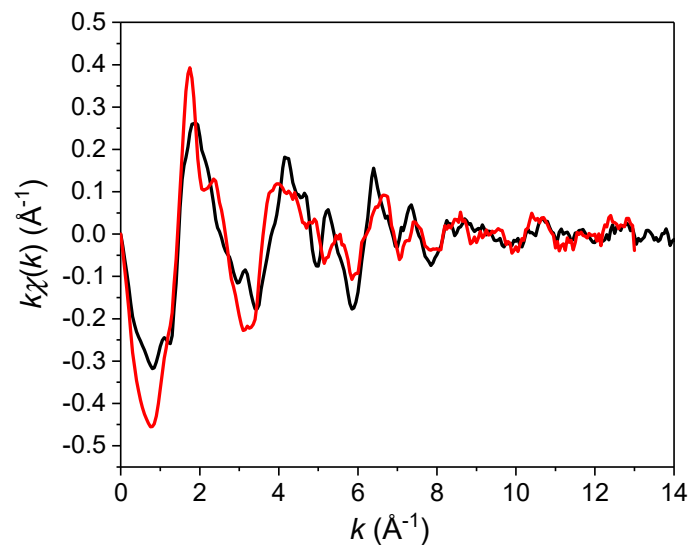

(a)

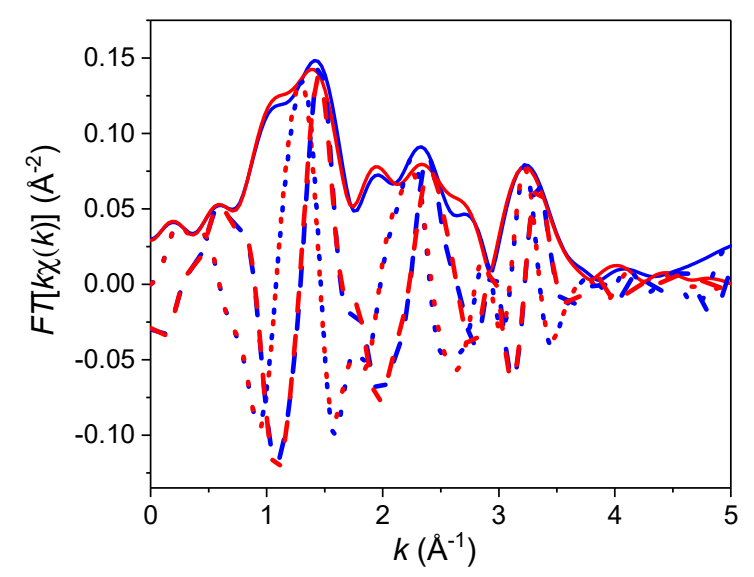

(b)

Figure 3. (a) Experimental $k$-weighted (where $k$ is the wavevector) EXAFS signal for the present $\mathrm{K}_{0.985} \mathrm{Mn}_{0.015} \mathrm{TaO}_{3 \pm \delta}(\mathrm{red})$ and previously analysed [29] $\mathrm{Sr}_{0.98} \mathrm{Mn}_{0.02} \mathrm{TiO}_{3}$ (black) ceramics. The latter composition was shown to contain a 0.6:0.4 mixture of $\mathrm{Mn}^{2+}$ and $\mathrm{Mn}^{4+}$ residing on the A- and B-sites, respectively. Note the overall similarity between the two signals, with differences accounted for by different backscattering species in the second- and higher-order coordination shells. (b) Experimental (blue) and fitted (red) Fourier transform (FT) of the EXAFS signal for $\mathrm{K}_{0.985} \mathrm{Mn}_{0.015} \mathrm{TaO}_{3 \pm \delta}$. The magnitude of the FT is shown using solid lines, whereas the real and imaginary parts of the FT are indicated using dashed and dotted lines, respectively. The $k$ range used in the FT was $2.68 \AA^{-1}$ to $10.98 \AA^{-1}$. The $R$ factor describing the quality of the fit is 0.0095 .

Model 2 provided a poor fit to the data, especially in the $r$-range with strong multiplescattering contributions, which are characteristic of the octahedral coordination in perovskites. Additionally, some of the refined structural variables acquired unphysical values. Therefore, we discarded this model. In contrast, Model 1 reproduced the data satisfactorily with sound values for the parameters (Table 2), which overall were consistent with the features of $\mathrm{Mn}^{2+}$ in the cuboctahedral coordination reported previously for Mn-doped ST and CT. The refined fraction of the cuboctahedral Mn was $0.73 \pm 0.04$, consistent with the 
estimate for the $\mathrm{Mn}^{2+}$ species from the linear combination fit of the XANES. As observed in other perovskite compounds, the relatively small $\mathrm{Mn}^{2+}$ cations (ionic radius $\approx 1.27 \AA$ in the cuboctahedral coordination [30]) are significantly off-centred within the K-site oxygen cages, forming several short Mn-O bonds. The refined values of the D-W factors indicate a significant spread even for such short distances. Additionally, the $\mathrm{Mn}^{2+}$-Ta distance appears to be shorter than expected from the average KT structure, suggesting a relaxation of the Ta cations around the K-site Mn. We feel, however, that the present EXAFS data are too limited for a more detailed understanding of structural relaxations induced by $\mathrm{Mn}$.

Table 2. Parameters of the Mn coordination environment in $\mathrm{K}_{0.985} \mathrm{Mn}_{0.015} \mathrm{TaO}_{3 \pm \delta}$ as obtained by fitting the EXAFS data ( $N$ coordination numbers; $R$, interatomic distances; $\sigma^{2}$, Debye-Waller factors). In these fits, the amplitude-reduction factor was set at 1 , as predicted by the FEFF calculations, and $\mathrm{E}_{0}$ at the energy of the 2nd pre-edge feature (Figure 2). The uncertainties (a single standard deviation) in the refined parameters, as calculated by the Artemis software, are specified in parentheses.

\begin{tabular}{ccccc}
\hline Valence (Site) & Shell & $\boldsymbol{N}$ & $\boldsymbol{R}(\AA)$ & $\sigma^{2}\left(\AA^{2}\right)$ \\
\hline \multirow{3}{*}{$\mathrm{Mn}^{4+}$ (Ta site) } & $\mathrm{Mn}-\mathrm{O}$ & 6 & $1.9^{\mathrm{a}}$ & $0.005^{\mathrm{a}}$ \\
& $\mathrm{Mn}-\mathrm{K}$ & 8 & $3.34(6)$ & $0.009^{\mathrm{a}}$ \\
& $\mathrm{Mn}-\mathrm{Ta}$ & 6 & $3.88^{\mathrm{a}}$ & $0.009^{\mathrm{a}}$ \\
\hline \multirow{3}{*}{$\mathrm{Mn}^{2+}(\mathrm{K}$ site) } & $\mathrm{Mn}-\mathrm{O}$ & 3 & $2.34(5)$ & $0.027(1)$ \\
& & 6 & $2.86(7)$ & $0.041^{\mathrm{b}}$ \\
& $\mathrm{Mn}-\mathrm{Ta}$ & 3 & $3.4^{\mathrm{a}}$ & $0.054^{\mathrm{b}}$ \\
& $\mathrm{Mn}-\mathrm{K}$ & 6 & $3.23(2)$ & $0.008(2)$ \\
& 6 & $3.96(2)$ & $0.019(5)$ \\
\hline
\end{tabular}

a These parameters were kept fixed. ${ }^{\mathrm{b}}$ These values of $\sigma^{2}$ were defined as $\sigma_{2}{ }^{2}(\mathrm{Mn}-\mathrm{O})=1.5 \sigma_{1}{ }^{2}(\mathrm{Mn}-\mathrm{O})$ and $\sigma_{3}^{2}(\mathrm{Mn}-\mathrm{O})=2 \sigma_{1}{ }^{2}(\mathrm{Mn}-\mathrm{O})$, where $\sigma_{1}, \sigma_{2}$, and $\sigma_{3}$ are $\mathrm{D}-\mathrm{W}$ factors for the three $\mathrm{Mn}(\mathrm{A})$-O distances (short, medium, long) used in the model.

Interestingly, we obtained similar XANES and EXAFS for $\mathrm{KTa}_{0.985} \mathrm{Mn}_{0.015} \mathrm{O}_{3 \pm \delta}$ ceramics synthesized under the conditions identical to those described in the experimental section. This similarity points to an amphoteric behaviour of Mn dopants in KT, with the preferred oxidation states and site occupancies being independent of Mn substitution for $\mathrm{K}$ or Ta in the chemical formula. This behaviour resembles that recently reported for $\mathrm{Mn}$ in $\mathrm{CT}$. In the latter system, it was attributed to octahedral rotations which stabilize $\mathrm{Mn}^{2+}$ on the cuboctahedral sites [6]. In KT, the preferential occupancy of $\mathrm{Mn}^{2+}$ on the K-site can be promoted by the difficult-to-avoid $\mathrm{K}$ deficiency caused by the volatility of these species.

In the case of Li doping, the dielectric relaxations induced by off-centre displacements of $\mathrm{Li}^{+}$ions on $\mathrm{K}$ sites are accompanied by a ferroelectric phase transition $[8,9,13]$. This transition is reflected in the split TO1 mode in low-temperature Raman spectra for the $\mathrm{Li}$ content greater than $1.4 \%$ [31], as shown by arrows in Figure 4a for $\mathrm{K}_{0.98} \mathrm{Li}_{0.02} \mathrm{TaO}_{3}$ ceramics [12]. In contrast, for the present $\mathrm{K}_{0.985} \mathrm{Mn}_{0.015} \mathrm{TaO}_{3 \pm \delta}$ ceramics, the spectra display no pronounced TO1 mode, as also observed for undoped KT [12]. At room temperature, all three compositions yield similar spectra (Figure 4b).

Figure 4c illustrates the evolution of spectra for the Mn-doped KT ceramics as a function of temperature. First-order Raman scattering in both undoped and Mn-doped $\mathrm{KT}$ is forbidden by their cubic Pm-3m symmetry so that most of the features seen in Figure 4 arise from second-order Raman processes. However, the broad features around 50 (labelled as TA) and $110 \mathrm{~cm}^{-1}$ (2TA) that are visible in the room-temperature spectra but gradually disappear on cooling are related to the two-phonon scattering linked to a peak in the density of states of the transverse acoustic (TA) branch at the Brillouin zone boundary [31,32]. Several Raman features emerge on cooling, including those from the infrared-active optical modes around $547 \mathrm{~cm}^{-1}$ (TO4) and $200 \mathrm{~cm}^{-1}$ (TO2), as well as a broad, low-frequency shoulder assigned to the TO1 mode [32]. The appearance of these optical modes in ceramics has been attributed to frozen electric dipoles at grain boundaries, 
resulting in the local loss of inversion symmetry, thus breaking the selection rules for

Raman and infrared activities [33].
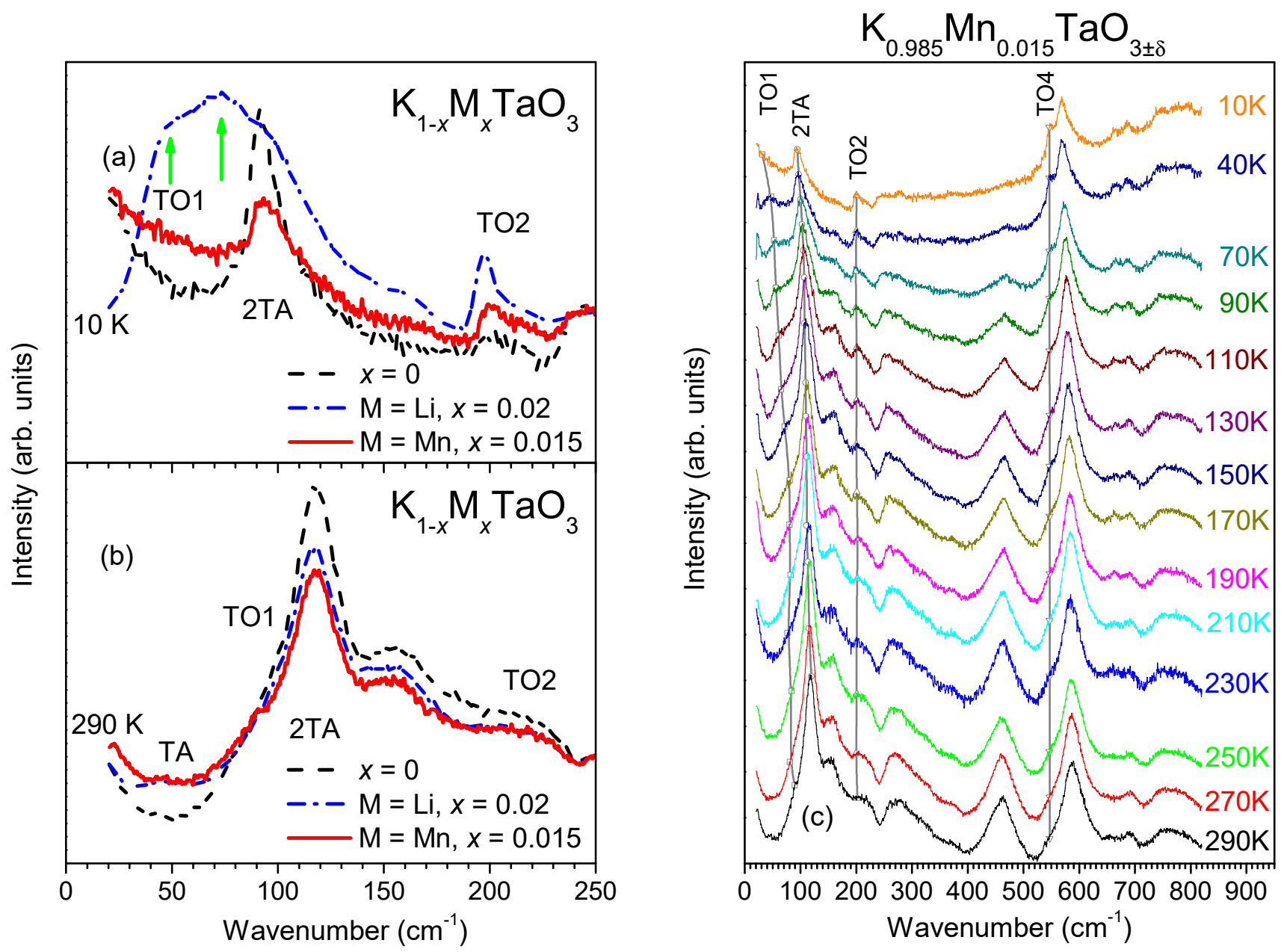

Figure 4. (a,b) Lower-wavenumber portions of Raman spectra for $\mathrm{K}_{0.985} \mathrm{Mn}_{0.015} \mathrm{TaO}_{3 \pm \delta}$ ceramics (solid lines) at $10 \mathrm{~K}$ (a) and $290 \mathrm{~K}$ (b) compared to those of ceramic $\mathrm{KTaO}_{3}$ (dash lines) and $\mathrm{K}_{0.98} \mathrm{Li}_{0.02} \mathrm{TaO}_{3}$ (dash dot lines) recorded at the same temperatures. (c) Raman spectra of the $\mathrm{K}_{0.985} \mathrm{Mn}_{0.015} \mathrm{TaO}_{3 \pm \delta}$ ceramics over a broad wavenumber range recoded between $10 \mathrm{~K}$ and $290 \mathrm{~K}$ (c).

We fitted the first-order features in the background-subtracted Raman spectra using a model of independent damped oscillators [12]. According to this model, the Raman intensity $I(\omega, T)$ is described as

$$
I(\omega, T)=(1+n(\omega, T)) \sum_{j=1}^{N}\left(A_{o j} \frac{\omega \Omega_{o j}^{2} \Gamma_{o j}}{\left(\Omega_{o j}^{2}-\omega^{2}\right)^{2}+\omega^{2} \Gamma_{o j}^{2}}\right)
$$

where $\omega$ is the frequency, $T$ is the temperature, $n(\omega, T)$ is the Bose-Einstein factor, and $A_{o j}$, $\Omega_{o j}$, and $\Gamma_{o j}$ are the strength, the wavenumber, and the damping coefficient for the $j$-th oscillator, respectively. The fitted frequencies of the TO1, TO2, TO4, and 2TA modes for the doped ceramics are plotted in Figure 5 as a function of temperature. While frequencies of the TO4 and TO2 modes remain approximately constant, the TO1 mode softens continuously upon cooling, resembling the behaviour of this mode in undoped KT. Below $80 \mathrm{~K}$, the frequency of TO1 for the doped ceramics $\left(32 \mathrm{~cm}^{-1} @ 10 \mathrm{~K}\right)$ appears to be higher than that for KT $\left(23 \mathrm{~cm}^{-1} @ 10 \mathrm{~K}\right)$, which is in agreement with the lower dielectric permittivity of the former if estimated according to the Lyddane-Sachs-Teller relation, $\Delta \varepsilon_{j} \Omega_{T O j}^{2}=$ const [34]. 


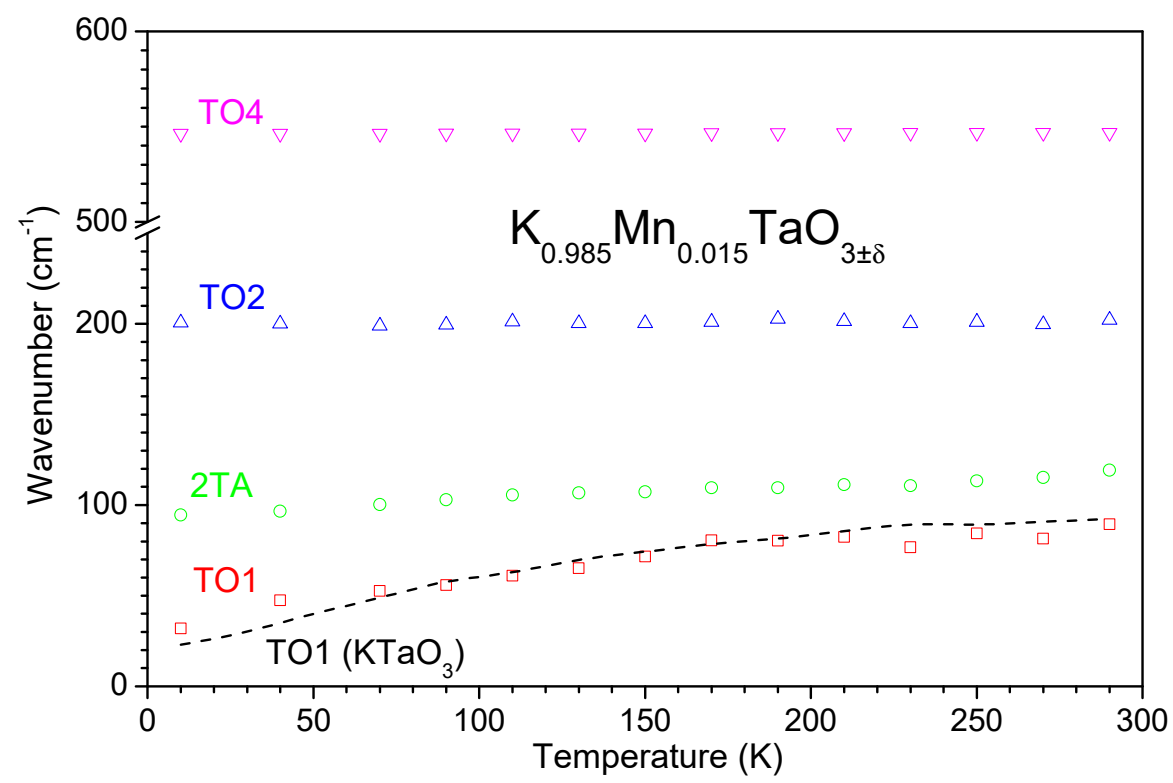

Figure 5. Variation of the TO1 (squares), 2TA (circles), TO2 (up triangles), and TO4 (down triangles) Raman modes in the $\mathrm{K}_{0.985} \mathrm{Mn}_{0.015} \mathrm{TaO}_{3 \pm \delta}$ ceramics with temperature. The behaviour of the TO1 mode for undoped KT ceramics (dashed line) is shown for comparison.

The appearance of the TO1 feature involving the mode hardening on cooling for Mn-doped KT relative to the undoped compound resembles the behaviour of this mode in ST with 2.5\% Mn substituted on the Sr site [30]. In contrast, for the Mn substitution on the Ti-site, the frequency of the TO1 mode was higher than that in undoped ST over the entire temperature range. Thus, the temperature behaviour of the TO1 mode in the present ceramics is consistent with $\mathrm{Mn}$ residing on the cuboctahedral sites, as suggested by our XAFS analysis. At the same time, the differences between the behaviour of the TO1 mode in Mn and Li doped KT indicate that while both species reside on the K sites and exhibit significant polar displacements, the coupling of such displacements to the host lattice is weaker for $\mathrm{Mn}$, resulting in dielectric relaxation with no ferroelectric transition.

\section{Conclusions}

Mn-doped KT ceramics exhibit a pronounced dielectric relaxation with the Arrheniuslaw parameters $U \approx 105 \mathrm{meV}$ and $\tau_{0} \approx 4.6 \times 10^{-14} \mathrm{~s}$. This behaviour is attributed to a displacive disorder of the $\mathrm{Mn}^{2+}$ species occupying the oversized cuboctahedral K sites, as demonstrated using analyses of the X-ray absorption fine structure for the Mn K-edge and variable-temperature Raman spectroscopy. In the Raman spectra, the presence of polar Mn off-centring and the coupling of the resulting electric dipoles to the host lattice is manifested by the hardening of the optical, low-frequency TO1 mode at low temperatures relative to the undoped $\mathrm{KT}$. A comparison with $\mathrm{Li}$-doped $\mathrm{KT}$, where $\mathrm{Li}^{+}$cations also occupy $\mathrm{K}$ sites and exhibit polar displacements, indicates that the strength of such a coupling for $\mathrm{Mn}$ is weaker, yielding dielectric relaxation but without triggering a ferroelectric transition as observed in the Li case.

Author Contributions: Conceptualization, A.T.; methodology, A.T. and A.A.; validation, A.T. and I.L.; formal analysis, A.T., A.A., I.L. and P.M.V.; investigation, A.T.; resources, P.M.V. and A.A.; data curation, A.T., A.A., J.C.W. and I.L.; writing—original draft preparation, A.T.; writing—review and editing, A.T., A.A., J.C.W. and I.L.; visualization, A.T. and I.L.; funding acquisition, A.A. and P.M.V. All authors have read and agreed to the published version of the manuscript.

Funding: This work was partially developed within the scope of the project CICECO-Aveiro Institute of Materials, UIDB/50011/2020 \& UIDP/50011/2020, financed by national funds through the Portuguese Foundation for Science and Technology/MCTES. 
Institutional Review Board Statement: Not applicable.

Informed Consent Statement: Not applicable.

Data Availability Statement: The data presented in this study are available on request from the corresponding author.

Conflicts of Interest: The authors declare no conflict of interest.

\section{References}

1. Lemanov, V.V.; Sotnikov, A.V.; Smirnova, E.P.; Weihnacht, M.; Kunze, R. Perovskite $\mathrm{CaTiO}_{3}$ as an incipient ferroelectric. Solid State Commun. 1999, 110, 611-614. [CrossRef]

2. Geyer, R.G.; Riddle, B.; Krupka, J.; Boatner, L.A. Microwave dielectric properties of single-crystal quantum paraelectrics $\mathrm{KTaO}_{3}$ and $\mathrm{SrTiO}_{3}$ at cryogenic temperatures. J. Appl. Phys. 2005, 97, 104111. [CrossRef]

3. Tagantsev, A.K.; Sherman, V.O.; Astafiev, K.F.; Venkatesh, J.; Setter, N. Ferroelectric materials for microwave tunable applications. J. Electroceram. 2003, 11, 5-66. [CrossRef]

4. Davis, T.G. Dielectric properties and soft modes in the ferroelectric mixed crystals $\mathrm{K}_{1-x} \mathrm{Na}_{x} \mathrm{TaO}_{3}$. Phys. Rev. B 1972, 5, $2530-2537$. [CrossRef]

5. Maier, R.A.; Cockayne, E.; Donohue, M.; Cibin, G.; Levin, I. Substitutional mechanisms and structural relaxations for manganese in $\mathrm{SrTiO}_{3}$ : Bridging the concentration gap for point-defect metrology. Chem. Mater. 2020, 32, 4651-4662. [CrossRef]

6. Maier, R.A.; Garrity, K.; Ozarowski, A.; Donohue, M.P.; Cibin, G.; Levin, I. Effects of octahedral tilting on the site of substitution of manganese in $\mathrm{CaTiO}_{3}$. Acta Mater. 2021, 207, 1116688. [CrossRef]

7. Kleemann, W.; Dec, J.; Tkach, A.; Vilarinho, P.M. SrTiO 3 -glimpses of an inexhaustible source of novel solid state phenomena. Condens. Matter 2020, 5, 58. [CrossRef]

8. Samara, G.A. The relaxational properties of compositionally disordered $\mathrm{ABO}_{3}$ perovskites. J. Phys. Condens. Matter 2003, 15, R367-R411. [CrossRef]

9. Vugmeister, B.E.; Glinchuk, M.D. Dipole glass and ferroelectricity in random-site electric dipole systems. Rev. Mod. Phys. 1990, 62, 993-1026. [CrossRef]

10. Tkach, A.; Vilarinho, P.M. Nonstoichiometry Role on the Properties of Quantum-Paraelectric Ceramics. In Structure Processing Properties Relationships in Stoichiometric and Nonstoichiometric Oxides; Tanasescu, S., Ed.; IntechOpen: London, UK, 2020.

11. Tkach, A.; Almeida, A.; Agostinho Moreira, J.; Espinha, A.; Chaves, M.R.; Perez de la Cruz, J.; Vilarinho, P.M. Lithium-induced dielectric relaxations in potassium tantalate ceramics. J. Phys. D Appl. Phys. 2011, 44, 315406. [CrossRef]

12. Tkach, A.; Almeida, A.; Agostinho Moreira, J.; Chaves, M.R.; Espinha, A.; Vilarinho, P.M. Polar behaviour induced by lithium in potassium tantalate ceramics. J. Phys. Condens. Matter 2012, 24, 045906. [CrossRef]

13. Trybula, Z.; Dec, J.; Miga, S.; Los, S.; Trybula, M. Coexistence of the relaxor-like and ferroelectric behavior in $\mathrm{K}_{1-\mathrm{x}} \mathrm{Li}_{\mathrm{x}} \mathrm{TaO}_{3}$. Phase Transit. 2016, 89, 794-802. [CrossRef]

14. Nowick, A.S.; Fu, S.Q.; Lee, W.-K.; Lim, B.S.; Scherban, T. Dielectric relaxation of paired defects in perovskite-type oxides. Mater. Sci. Eng. B 1994, 23, 19-24. [CrossRef]

15. Laguta, V.V.; Glinchuk, M.D.; Bykov, I.P.; Rosa, J.; Jastrabik, L.; Savinov, M.; Trybuła, Z. Paramagnetic dipole centers in KTaO 3 : Electron-spin-resonance and dielectric spectroscopy study. Phys. Rev. B 2000, 61, 3897-3904. [CrossRef]

16. Venturini, E.L.; Samara, G.A.; Laguta, V.V.; Glinchuk, M.D.; Kondova, I.V. Dipolar centers in incipient ferroelectrics: Mn and Fe in $\mathrm{KTaO}_{3}$. Phys. Rev. B 2005, 71, 094111. [CrossRef]

17. Kleemann, W.; Bedanta, S.; Borisov, P.; Shvartsman, V.V.; Miga, S.; Dec, J.; Tkach, A.; Vilarinho, P.M. Multiglass order and magnetoelectricity in $\mathrm{Mn}^{2+}$ doped incipient ferroelectrics. Eur. Phys. J. B 2009, 71, 407-410. [CrossRef]

18. Shvartsman, V.V.; Bedanta, S.; Borisov, P.; Kleemann, W.; Tkach, A.; Vilarinho, P.M. Spin cluster glass and magnetoelectricity in Mn-doped $\mathrm{KTaO}_{3}$. J. Appl. Phys. 2010, 107, 103926. [CrossRef]

19. Axelsson, A.-K.; Pan, Y.; Valant, M.; Vilarinho, P.M.; Alford, N.M. Polar fluctuations in Mn substituted KTaO 3 ceramics. J. Appl. Phys. 2010, 108, 064109. [CrossRef]

20. Axelsson, A.-K.; Pan, Y.; Valant, M.; Alford, N. Chemistry, processing, and microwave dielectric properties of Mn-substituted $\mathrm{KTaO}_{3}$ ceramics. J. Am. Ceram. Soc. 2010, 93, 800-805. [CrossRef]

21. Axelsson, A.-K.; Valant, M.; Alford, N.M. Influence of point defects in $\mathrm{KTaO}_{3}$ on low-temperature dielectric relaxation. J. Eur. Ceram. Soc. 2010, 30, 941-946. [CrossRef]

22. Tkach, A.; Vilarinho, P.M.; Kleemann, W.; Shvartsman, V.V.; Borisov, P.; Bedanta, S. Comment on "The origin of magnetism in Mn-doped $\mathrm{SrTiO}_{3} "$. Adv. Funct. Mater. 2013, 23, 2229-2230. [CrossRef]

23. Tkach, A.; Vilarinho, P.M.; Almeida, A. Microscopy and the solid solubility limit in $\mathrm{K}_{1-x} \mathrm{Mn}_{x} \mathrm{TaO}_{3}$ ceramics. Microsc. Microanal. 2012, 18 (Suppl. 5), 89-90. [CrossRef]

24. Valant, M.; Kolodiazhnyi, T.; Axelsson, A.-K.; Babu, G.S.; Alford, N.M. Spin ordering in Mn-doped KTaO3 ${ }_{3}$ Chem. Mater. 2010, 22, 1952-1954. [CrossRef] 
25. Woicik, J.C.; Ravel, B.; Fischer, D.A.; Newburgh, W.J. Perofrmance of a four-element Si drift detector for X-ray absorption fine-structure spectroscopy: Resolution, maximum count rate, and dead-time correction with incorporation into the ATHENA data analysis software. J. Syncrhotron Radiat. 2010, 17, 409-413. [CrossRef] [PubMed]

26. Ravel, B.; Newville, M. ATHENA, ARTEMIS, HEPHAESTUS: Data analysis for X-ray absorption spectroscopy using IFEFFIT. J. Synchrotron Radiat. 2005, 12, 537-541. [CrossRef] [PubMed]

27. Rehr, J.J.; Kas, J.J.; Prange, M.P.; Sorini, A.P.; Takimoto, Y.; Vila, F. Ab initio theory and calculations of X-ray spectra. Comptes Rendus Phys. 2009, 10, 548-559. [CrossRef]

28. Shannon, R.D. Revised effective ionic radii and systematic studies of interatomic distances in halides and chalcogenides. Acta Crystallogr. Sect. A 1976, 32, 751-767. [CrossRef]

29. Levin, I.; Krayzman, V.; Woicik, J.C.; Tkach, A.; Vilarinho, P.M. X-ray absorption fine structure studies of Mn coordination in doped perovskite $\mathrm{SrTiO}_{3}$. Appl. Phys. Lett. 2010, 96, 052904. [CrossRef]

30. Tkach, A.; Vilarinho, P.M.; Nuzhnyy, D.; Petzelt, J. Sr- and Ti-site substitution, lattice dynamics, and octahedral tilt transition relationship in $\mathrm{SrTiO}_{3}: \mathrm{Mn}$ ceramics. Acta Mater. 2010, 58, 577-582. [CrossRef]

31. Prater, R.L.; Chase, L.L.; Boatner, L.A. Raman scattering studies of the impurity-induced ferroelectric phase transition in $\mathrm{KTaO}_{3}: \mathrm{Li}_{\text {. }}$ Phys. Rev. B 1981, 23, 5904-5915. [CrossRef]

32. Prater, R.L.; Chase, L.L.; Boatner, L.A. Raman scattering studies of the impurity-induced ferroelectric phase transition in $\mathrm{KTaO}_{3}: \mathrm{Nb}$. Phys. Rev. B 1981, 23, 221-231. [CrossRef]

33. Petzelt, J.; Ostapchuk, T. Infrared and Raman spectroscopy of some ferroelectric perovskite films and ceramics. J. Optoelectron. Adv. Mater. 2003, 5, 725-733.

34. Lyddane, R.H.; Sachs, R.G.; Teller, E. On the polar vibrations of alkali halides. Phys. Rev. 1941, 59, 673-676. [CrossRef] 